\title{
Efficacy of Precesarean Infrarenal Abdominal Aorta Balloon Occlusion Among Women With Pernicious Placenta Previa and Placenta Accreta: A Retrospective Cohort Analysis
}

\section{Xuemin Wei}

International Peace Maternity and Child Health Hospital

Yan Chen

International Peace Maternity and Child Health Hospital

Weiwei Cheng ( $\nabla$ wwcheng29@shsmu.edu.cn )

International Peace Maternity and Child Health Hospital https://orcid.org/0000-0002-0805-8296

\section{Research Article}

Keywords: pernicious placenta previa, placenta accreta, prophylactic balloon occlusion of the infrarenal abdominal aorta, cesarean section

Posted Date: October 18th, 2021

DOl: https://doi.org/10.21203/rs.3.rs-919396/v1

License: (c) (1) This work is licensed under a Creative Commons Attribution 4.0 International License.

Read Full License 


\section{Efficacy of Precesarean Infrarenal Abdominal Aorta Balloon Occlusion among Women with Pernicious Placenta Previa and Placenta Accreta: A retrospective cohort analysis}

Xuemin Wei, Yan Chen, Weiwei Cheng

\section{First Author: Xuemin Wei}

International Peace Maternal and Child Health Hospital, Shanghai, China, address information :910 Hengshan Road, Xuhui District, Shanghai, China

Email: weixuemin0539@163.com

\section{Second Author: Yan Chen}

International Peace Maternal and Child Health Hospital, Shanghai, China, address information :910 Hengshan Road, Xuhui District, Shanghai, China

Email: 507595623@qq.com

\section{Corresponding author: Weiwei Cheng}

International Peace Maternal and Child Health Hospital, Shanghai, China, address information :910 Hengshan Road, Xuhui District, Shanghai, China Email: wwcheng29@shsmu.edu.cn 


\begin{abstract}
Purpose To evaluate the efficacy and safety of prophylactic balloon occlusion of the infrarenal abdominal aorta among women with pernicious placenta previa and placenta accreta.

Methods This retrospective study included 110 patients with pernicious placenta previa and placenta accreta. The control group consisted of 55 patients who underwent cesarean section alone, and the study group included 55 patients who underwent precesarean prophylactic balloon occlusion of the infrarenal abdominal aorta. In addition, both of the groups were further divided according to FIGO clinical grading standards. Prevention of hysterectomy was the primary outcome evaluated. The secondary outcomes included operative duration, estimated blood loss, blood transfusion, intensive care unit admission, total hospital stay (days), and puerperal morbidity, and these data were compared between the two groups. Additionally, the neonatal outcomes were compared.
\end{abstract}

Results There were no significant differences in maternal and neonatal outcomes in the PAS 2 and PAS 3 groups (P > 0.05). However, in the PAS 4 and PAS 5 groups, the amount of bleeding in the study group was significantly less than that in the control group $(3533.3 \pm 2391.4$ vs $4293.6 \pm 1235.4, \mathrm{P}<0.05)$, and the total hysterectomy rate was also lower $(7.8 \%$ vs $13.2 \%, \mathrm{P}<0.05)$.

Conclusion Precesarean infrarenal abdominal aortic balloon occlusion is an effective and safe option for treating pernicious placenta previa and placenta accreta and can effectively reduce the risk of hysterectomy and intraoperative blood loss in women with PAS grade 4-5.

Key words: pernicious placenta previa; placenta accreta; prophylactic balloon occlusion of the infrarenal abdominal aorta; cesarean section 


\section{Introduction}

Pernicious placenta previa (PPP), a condition in which the placenta attaches to a previous surgical scar site, is often related to placenta accreta. With the implementation of the three-child policy in China, the proportion of pregnant women with a previous history of cesarean section has increased[1,2], which has led to an increase in the incidence of pernicious placenta previa. It has been reported that a history of previous cesarean section and placenta previa are the most common high-risk factors for placenta accreta[3]; other risk factors include advanced maternal age, multiple induced abortions and in vitro fertilization[4,5]. Placenta accreta often occurs in the lower segment of the anterior wall of the uterus and is related to abnormal placental invasion caused by secondary loss of endometrial-myometrial junctions[6]. Therefore, pernicious placenta previa is often associated with placenta accreta, which has become an important cause of refractory postpartum hemorrhage, hemorrhagic shock, diffuse intravascular coagulation (DIC) and perinatal emergency hysterectomy, seriously threatening the life and safety of the mother and fetus[7,8].

With the widespread application of vascular interventional therapy in obstetrics in recent years, women with pernicious placenta previa and placenta accreta have undergone balloon occlusion of the abdominal aorta (BOAA) before cesarean section[9,10]. However, the efficacy of this 
technique is not clear at present. In this paper, the effect of precesarean balloon occlusion of the abdominal aorta on maternal and fetal prognosis was retrospectively analyzed, and its effectiveness and safety were evaluated.

\section{Materials and Methods}

\subsection{Patients}

We performed a retrospective study of patients with pernicious placenta previa with concomitant placenta accreta at International Peace Maternal and Child Health Hospital Affiliated with Shanghai Jiaotong University, China, between January 2014 and December 2020. Patients were included if they met the following criteria: (1) Pernicious placenta previa with placenta accreta diagnosed by color Doppler ultrasound and/or MRI examination before cesarean section. (2) Definitive diagnosis that met the clinical description during cesarean section. (3) A history of at least one previous CS without other obstetric diseases. According to the 2018 FIGO guidelines[11], clinical description is the most important standard for the diagnosis and classification of PAS; therefore, the postoperative placental pathological diagnosis is used only as a reference standard in this study.

Finally, a total of 110 patients were included in this study. Since 2016, our hospital has used balloon implantation in the abdominal aorta. The 55 patients comprising the study group underwent abdominal aortic balloon placement before cesarean section, and 55 patients treated before 2016 who 
did not undergo abdominal aortic balloon placement comprised the control group. The patients were further divided into several groups according to the PAS clinical grading standard in the FIGO guidelines of 2018. All of the patients provided informed consent. This study was approved by the ethical committee of International Peace Maternal and Child Health Hospital Affiliated with Shanghai Jiaotong University, China.

\section{Methods}

2.1 Preoperative preparation:

(1) The whole-body condition of the pregnant women and the intrauterine condition of the fetuses were comprehensively evaluated before the operation, and the degree of placental accreta was determined by color Doppler ultrasound and/or magnetic resonance imaging. Multidisciplinary cooperation included obstetrics, gynecology, neonatal pediatrics, interventional radiology, blood transfusion, maternal and fetal critical medicine, anesthesiology, imaging, urology and other preoperative and intraoperative consultations if necessary. (2) All patients were fully informed of the risk_before the operation and signed the informed consent form for the operation. (3) Blood and autotransfusion equipment were fully prepared. (4) Venous access was established, including peripheral and central venous catheterization.

2.1 Operation process: (1) Study group: The women were treated with balloon occlusion of the infrarenal abdominal aorta by an experienced 
interventional radiologist in a digital subtraction angiography (DSA) room before CS. After local anesthesia was administered, the right femoral artery was punctured using the Seldinger technique. An $8 \mathrm{~F}$ catheter sheath was inserted, and a $4 \mathrm{f}$ pigtail catheter was introduced and sent to the L1 level of the abdominal aorta. The left and right common iliac bifurcations were located at the level of L4. The pigtail catheter was withdrawn. Under the guidance of a 0.035 guidewire, a $40 * 16 \mathrm{~mm}$ balloon catheter was sent to the upper part of L1. After the guidewire was removed and heparin was injected, the three-way tube was connected, and the other balloon interface was connected with a pressure pump. After the pressure reached $4 \mathrm{~Pa}$, the balloon was completely expanded, and the abdominal aorta was blocked gradually. The X-ray exposure field per square meter was $10 \mathrm{mGy}$, and the exposure time was 2-2.5 minutes. After the operation was successfully performed, the patient was sent to the operating room. (2) During cesarean section, after the fetus was delivered and the umbilical cord was clipped, normal saline was injected into the balloon to block the blood flow of the abdominal aorta. Placental dissection and uterine suture were performed. If there was active bleeding during the operation, the balloon was released every 15 minutes after 1 minute of filling to restore the blood flow of the abdominal aorta. The obstetricians decided whether other surgical methods were needed according to the intrapartum hemorrhage and placenta accreta situation; possible additional surgical methods included local continuous 
figure- 8 suturing of the uterine wound, B-Lynch suturing of the uterus, transverse ring or butterfly suturing of the lower uterine segment, intrauterine water sac packing, uterine artery ligation, etc. If the bleeding was still uncontrollable, hysterectomy was performed. If the balloon pressure was released during the operation and there was no active bleeding, the balloon was kept released until the end of the operation. The balloon was released when the uterine incision was sutured without bleeding and the maternal hemodynamics were stable. Uterine artery embolization was used for patients with persistent active bleeding. For those who required uterine artery embolization, the balloon catheter was withdrawn, a $4 \mathrm{f}$ uterine artery catheter was introduced, and an appropriate amount of gelatin sponge was injected into the opening of the left uterine artery until the disordered branches disappeared, and the blood flow was blocked on angiography; the right uterine artery was intubated, perfused and embolized in the same way. (3) After the cesarean section operation, the balloon catheter was removed, and a local pressure bandage was applied for 24 hours. Sensation, movement and dorsalis pedis artery pulsation of both lower limbs were observed after the operation. Antibiotics and oxytocin were used routinely after the operation, and low molecular weight heparin was injected subcutaneously 12 hours after the operation once a day until 1 week after delivery to prevent the occurrence of lower extremity venous thrombosis. 
(2) Control group: The control group comprised women who underwent CS to terminate a pregnancy. After delivery, uterine artery ligation or uterine cavity filing with a Bakri balloon tamponade or ribbon gauze was performed when hemorrhaging did not stop. If hemorrhaging could not be controlled, a hysterectomy was performed.

In all cases, pathological examination of the placenta was performed.

\section{Outcomes}

The primary outcome was the prevention of hysterectomy. The secondary outcomes were duration of the operation; intraoperative blood loss; intraoperative blood transfusion volume; postoperative changes in hemoglobin level; postoperative intensive care unit (ICU) admission; postoperative hospital stay; postoperative morbidity, such as bladder injury and infection; and duration of postoperative antibiotic use. Neonatal outcomes, including 1- and 5-minute low Apgar scores and birth weight, were also evaluated for the two groups.

\section{Statistical methods}

SPSS.26 software was used to process the data. Quantitative data are expressed as the mean \pm standard deviation, and comparisons between groups were made using independent sample t-tests or nonparametric tests; count data are expressed as rates, and comparisons between groups were made using the chi square test or Fisher's exact probability method. Differences were considered statistically significant when $\mathrm{P}<0.05$. 


\section{Results}

Comparison of maternal demographic characteristics and the general situation between the two groups

One hundred ten women diagnosed with pernicious placenta previa with placenta accreta met the inclusion criteria and underwent CS. There were 55 cases in the study group and 55 in the control group. There was no significant difference in maternal age, gravity, parity, abortions, gestational weeks of delivery and previous number of cesarean sections/natural labors between the two groups $(\mathrm{P}>0.05)$, as shown in Table 1 . Therefore, there was no significant difference in clinical characteristics between the two groups, indicating that they were comparable.

\section{Comparison of the intraoperative situation between PAS grade groups}

The intraoperative situation of the two PAS grade groups was compared. For the PAS 2 and PAS 3 group, hysterectomy, duration of the operation, estimated blood loss, PPH (blood loss $\geq 1000 \mathrm{ml}$ ), postoperative decrease in the HGB level, transfusion rate, DIC rate, ICU admission, bladder repair, and maternal mortality were not significantly different between the study and the control groups, as shown in Table 2 .

For the PAS 4 and PAS 5 group, the rate of hysterectomy in the study group was significantly lower than that in the control group (15.38\% vs 63.64\%, $\mathrm{P}=0.03)$. The intraoperative blood loss in the study group was 
significantly less than that of the control group (2812.3 \pm 1756.7 vs 3204.4 $\pm 1565.1, \mathrm{P}=0.63$ ), with no statistical significance, and the rate of DIC in the study group was lower than that of the control group (38.46\% vs $81.82 \%, \mathrm{P}=0.05$ ), with no statistical significance, as shown in Table 2. There were no significant differences between the two groups in intraoperative assistive techniques, including intrauterine water sac packing, continuous figure-8 suturing and uterine artery embolization $(\mathrm{P}>$ 0.05), as shown in Table 2

Comparison of postoperation maternal and neonatal outcomes between the two groups

There were no significant differences in the postoperative hospitalization time, ICU stay, or maternal mortality measures, such as the thrombosis rate, between the two groups $(\mathrm{P}>0.05)$, as shown in Tables 2 . There were no significant differences in the neonates' birth weights or rate of low Apgar scores between the two groups $(\mathrm{P}>0.05)$, as shown in Table 3

\section{Discussion}

In the present study, we evaluated the efficacy and safety of precesarean infrarenal abdominal aorta balloon occlusion for the management of women with PPP with PA. Furthermore, we compared and analyzed the women by different PAS grades based on the FIGO grading standard of 2018, as shown in Table 6. We found that for the PAS 2 and 
PAS 3 group, there was no significant difference between the study and control groups in terms of hysterectomy, duration of operation, estimated blood loss, blood transfusion, intensive care unit admission, puerperal morbidity or neonatal outcomes. For the PAS 4 and PAS 5 group, the rate of hysterectomy in the study group was significantly lower than that in the control group ( $15.38 \%$ vs $63.64 \%, \mathrm{P}=0.03)$. While the estimated blood loss and the incidence of DIC in the study group were lower than those in the control group $(2812.3 \pm 1756.7$ vs $3204.4 \pm 1565.1, \mathrm{P}=0.63 ; 38.46 \%$ vs $81.82 \%, \mathrm{P}=0.05)$, the difference was not significant.

The methods for controlling bleeding during cesarean section with PPP and PA include bilateral internal iliac artery or uterine artery ligation, intrauterine balloon compression, intrauterine gauze packing and various suture techniques. If bleeding is uncontrollable, immediate hysterectomy is often needed to ensure the patient's safety; however, this results in loss of the uterus and fertility. With the extensive application of radiation intervention technology during the perioperative period of cesarean section, abdominal aortic balloon implantation before cesarean section provides an opportunity for uterus and fertility preservation in patients with PPP and $\mathrm{PA}^{[12]}$.

Chen et al ${ }^{[10]}$ and $\mathrm{Wu}$ et al ${ }^{[13]}$ showed that abdominal aortic balloon implantation before cesarean section can effectively reduce intraoperative blood loss and the blood transfusion and hysterectomy rates. However, in 
this study, we did not find any significant difference in the amount of blood loss or the blood transfusion rate between the two groups.

$\mathrm{Na} \mathrm{Li} \mathrm{et} \mathrm{al}{ }^{[14]}$ showed that abdominal aortic balloon implantation prior to cesarean section can reduce the rate of hysterectomy in patients with PPP and PA, but it does not reduce the amount of bleeding or the proportion of blood transfusion. Additionally, $\mathrm{Hu}$ Rong et al. ${ }^{[15]}$ suggested that abdominal aortic balloon implantation can reduce the incidence of DIC and hysterectomy in cesarean section patients $(5.9 \%$ vs $34.8 \%, 17.6 \%$ vs $47.8 \%$, $\mathrm{P}<0.05$ ), but there is still a risk of continuous bleeding after balloon release, and other auxiliary methods are needed. There was no significant difference in the proportion of patients who required blood transfusions. A systematic review and meta-analysis demonstrated that the intraoperative blood loss volume, intraoperative blood transfusion volume, hysterectomy rate, operation time, postoperative hospitalization duration, and ICU admission rate were all reduced with the use of prophylactic AABO during CS in patients with PPP with PA ${ }^{[16]}$. In our study, based on FIGO guidelines, we conducted a further risk-grading study between the study group and the control group. Patients with PAS 4-5 pernicious placenta previa who underwent abdominal aortic balloon implantation before cesarean section had a significantly lower intraoperative blood loss volume $(3533.3 \pm 2391.4$ vs $4293.6 \pm 4, \mathrm{P}<0.05)$, and the total hysterectomy rate of the study group was lower than that of the control group (7.8\% vs $13.2 \%$, 
$\mathrm{P}<0.05)$. There were no significant differences in operation time, intraoperative blood loss, intraoperative blood transfusion, blood transfusion ratio, or preoperative and postoperative hemoglobin changes between the two groups $(\mathrm{P}>0.05)$. These findings suggest that preoperative evaluations can be performed according to the PAS clinical grading standards in the FIGO guidelines. Preoperative abdominal aortic balloon occlusion should be performed for patients with PAS 4-5 or above and can effectively reduce the volume of bleeding and the hysterectomy rate to achieve appropriate treatment.

Paull et al ${ }^{[17]}$ first used abdominal aortic balloon occlusion for hysterectomy with placenta accreta in 1995. Abdominal aorta balloon occlusion requires puncturing only one side of the femoral artery and inserting a balloon. After the temporary occlusion of the abdominal aorta, pelvic collateral circulation to uterine blood supply can be effectively controlled, temporarily reducing intraoperative blood loss, providing a clearer view for obstetricians, and facilitating follow-up operations, such as placental dissection and uterine suture. Additionally, the operation is relatively simple, which can shorten the corresponding operation time, reduce the radiation exposure of the mothers and fetuses, and block the blood supply of the pelvic cavity ${ }^{[18,19]}$. Studies have shown that in patients with PPP and PA, cesarean section can reduce the amount of bleeding to $1 / 10-1 / 5$ of the original ${ }^{[20]}$. The results of a systematic review by Shahin 
et al ${ }^{[21]}$ showed that the lowest blood loss volumes in cesarean section with pernicious placenta previa occurred when abdominal aorta balloon occlusion was used. In addition, the time required to place the balloon catheter in the abdominal aorta was the shortest, and the X-ray radiation dose received by the fetus was the lowest with abdominal aorta balloon occlusion.

The main risks and complications of abdominal aortic balloon implantation are drug allergy, bleeding (puncture point bleeding, internal bleeding caused by visceral vascular rupture), infection, aortic dissection, lower extremity thrombosis, etc. In this study, all abdominal aorta balloon implantation operations were completed by experienced chief physicians in the interventional radiology department. In the study group, the balloon was filled immediately after the delivery of the fetus, the blood flow of the abdominal aorta was blocked for 10-15 minutes, and the balloon was released for 1 minute before blocking. During and after the operation, the skin temperature and color of the lower limbs and the pulse of the dorsalis pedis artery were closely monitored. After the operation, the women were encouraged to exercise as soon as possible, wear elastic stockings and use low molecular weight heparin to prevent thrombosis of the lower limbs. There were no intraoperative or postoperative embolisms, arterial ruptures, cases of organ damage or maternal deaths in our study. There was no significant difference in the incidence of neonatal asphyxia or neonatal 
hospitalization rate between the two PAS groups. Considering the effect of radiation on the fetus, according to the regulations of the International Commission on radiation and protection, when the radiation dose is less than $100 \mathrm{mGy}$, the teratogenic risk to the fetus is not increased. In this study, the total fetal radiation exposure in the balloon occlusion group was less than $20 \mathrm{mGy}$, which was lower than the standard, indicating a low risk of fetal teratogenesis was lower.

In conclusion, preoperative abdominal aortic balloon occlusion has a clinical effect on the treatment of PPP with PA that provides a time window for conservative surgery. Before the operation, it is necessary to fully evaluate the MDT and strictly apply the indications for surgery. Other methods of obstetric hemostasis, such as uterine cavity filling, uterine artery embolization and various uterine suture techniques, can effectively reduce the bleeding and hysterectomy rate during cesarean section.

However, this study also has some limitations: (1) this was a retrospective study, the number of cases was small, and there may have been bias in case selection. Therefore, further large randomized controlled studies are needed to confirm the results. (2) In this study, the chief surgeon and the assistant performing the cesarean section were senior deputy chief physicians or above; however, the operating skills and experience of different obstetricians will differ, and the results with different operators need to be verified by further prospective multicenter studies with large 
samples in the future.

\section{Reference}

[1] Long Qian,Kingdon Carol,Yang Fan et al. Prevalence of and reasons for women's, family members', and health professionals' preferences for cesarean section in China: A mixed-methods systematic review.[J] .PLoS Med, 2018, 15: e1002672.

[2] Yan Jie, Wang Long, Yang Ying et al. The trend of caesarean birth rate changes in China after 'universal two-child policy' era: a population-based study in 2013-2018.[J] .BMC Med, 2020, 18: 249.

[3] Booker Whitney,Moroz Leslie,Abnormal placentation.[J] .Semin Perinatol, 2019, 43: 51-59. Coutinho C M, Giorgione V, Noel L et al. Effectiveness of contingent screening for placenta accreta spectrum disorders based on persistent low-lying placenta and previous uterine surgery.[J] .Ultrasound Obstet Gynecol, 2021, 57: 91-96.

[4] Kyozuka H, Yamaguchi A, Suzuki D, et al. Risk factors for placenta accreta spectrum: findings from the Japan environment and Children's study[J]. BMC Pregnancy Child. 2019;19(1):447.

[5] Salmanian Bahram, Fox Karin A, Arian Sara E et al. In vitro fertilization as an independent risk factor for placenta accreta spectrum.[J] .Am J Obstet Gynecol, 2020, 223: 568.e1-568.e5.

[6] Jauniaux Eric, Collins Sally, Burton Graham J, Placenta accreta spectrum: pathophysiology and evidence-based anatomy for prenatal ultrasound imaging. [J] .Am J Obstet Gynecol, 2018, 218: 75-87.

[7] Kassem GA, Ali A. Maternal and neonatal outcomes of placenta previa and placenta accreta: three years of experience with a two-consultant approach. Int J Women's Health. 2013:803

[8] Clinical Profiles of Placenta Accreta Spectrum: the PACCRETA Population-Based Study[J]. BJOG: An International Journal of Obstetrics \& Gynaecology, 2021.

[9] Li Kui,Zou Yu,Sun Jin et al. Prophylactic balloon occlusion of internal iliac arteries, common iliac arteries and infrarenal abdominal aorta in pregnancies complicated by placenta accreta: a retrospective cohort study.[J] .Eur Radiol, 2018, 28: 4959-4967.

[10] Chen Manru,Xie Lan,Clinical evaluation of balloon occlusion of the lower abdominal aorta in patients with placenta previa and previous cesarean section: A retrospective study on 43 cases.[J] .Int J Surg, 2016, 34: 6-9.

[11] Jauniaux Eric,Chantraine Frederic,Silver Robert M et al. FIGO consensus guidelines on 
placenta accreta spectrum disorders: Epidemiology.[J] .Int J Gynaecol Obstet, 2018, 140: 265-273.

[12] Huo Fei,Liang Hansheng,Feng Yi,Prophylactic temporary abdominal aortic balloon occlusion for patients with pernicious placenta previa: a retrospective study.[J] .BMC Anesthesiol, 2021, 21: 134.

[13] Wu Qinghua, Liu Zhuan, Zhao Xianlan et al. Outcome of Pregnancies After Balloon Occlusion of the Infrarenal Abdominal Aorta During Caesarean in 230 Patients With Placenta Praevia Accreta.

[J] .Cardiovasc Intervent Radiol, 2016, 39: 1573-1579.

[14] Li Na, Yang Tian, Liu Caixia et al. Feasibility of Infrarenal Abdominal Aorta Balloon Occlusion in Pernicious Placenta Previa Coexisting with Placenta Accrete.[J] .Biomed Res Int, 2018, 2018: 4596189.

[15] Hu Rong, Wu Wei, Wu Jiangnan et al., Clinical evaluation of prophylactic abdominal aorta balloon occlusion in patients with cesarean section of pernicious placenta previa and placenta accreta [J] Chinese Journal of Practical Gynecology and obstetrics,2018,34(8):902-906.

[16] He Qiang,Li Ying-Long,Zhu Ming-Juan et al. Prophylactic abdominal aortic balloon occlusion in patients with pernicious placenta previa during cesarean section: a systematic review and metaanalysis from randomized controlled trials.[J] .Arch Gynecol Obstet, 2019, 300: 1131-1145.

[17]Paull JD, Smith J, Williams L, et al.Balloon occlusion of the ab- dominal aorta during caesarean hysterectomy for placenta percreta $[\mathrm{J}]$.Anaesth Intensive Care, 1995, 23 (6) : 731734.

[18] Li Kui, Zou Yu, Sun Jin et al. Prophylactic balloon occlusion of internal iliac arteries, common iliac arteries and infrarenal abdominal aorta in pregnancies complicated by placenta accreta: a retrospective cohort study.[J] .Eur Radiol, 2018, 28: 4959-4967.

[19] Shahin Yousef, Pang Chun Lap, Endovascular interventional modalities for haemorrhage control in abnormal placental implantation deliveries: a systematic review and metaanalysis.[J] .Eur Radiol, 2018, 28: 2713-2726.

[20] Duan XH, Wang YL, Han XW, et al. Caesarean section combined with temporary aortic balloon occlusion followed by uterine artery embolisation for the management of placenta accrete[J]. Clin Radiol,2015,70:932-937.

[21] Shahin Y, Pang CL. Endovascular interventional modalities for haemorrhage control in abnormal placental implantation deliveries: A systematic review and meta-analysis[J]. Eur Radiol, 2018, 28(7): 2713-2726. 


\section{Author Contribution}

Xuemin Wei: Project development, Data Collection, statistical analysis, Manuscript writing

Yan Chen: Data collection

Weiwei Cheng: Project development, Manuscript writing

\section{First Author : Xuemin Wei}

International Peace Maternal and Child Health Hospital, Shanghai, China, address information :910 Hengshan Road, Xuhui District , Shanghai, China

Email :weixuemin0539@163.com

\section{Corresponding author : Weiwei Cheng}

International Peace Maternal and Child Health Hospital,Shanghai,China, address information :910 Hengshan Road, Xuhui District, Shanghai,China Communication Email: wwcheng29@shsmu.edu.cn 
Table 1: Comparison of maternal demographic and clinical characteristics

\begin{tabular}{lccc}
\hline & Study group $(\mathrm{n}=55)$ & Control group $(\mathrm{n}=55)$ & P value \\
\hline Maternal age $(\mathrm{y})$ & $36.6 \pm 4.1$ & $35.3 \pm 3.6$ & 0.08 \\
Gestational weeks $(\mathrm{wk})$ & $35.9 \pm 1.5$ & $36.1 \pm 2.1$ & 0.69 \\
Gravidity $(\mathrm{n})$ & $3.8 \pm 1.4$ & $3.6 \pm 1.3$ & 0.45 \\
Parity $(\mathrm{n})$ & $2.1 \pm 0.4$ & $2.1 \pm 0.3$ & 0.24 \\
Prior abortions(n) & $1.6 \pm 1.3$ & $1.5 \pm 1.3$ & 0.67 \\
Previous cesarean delivery $(\mathrm{n})$ & $1.1 \pm 0.4$ & $1.0 \pm 0.1$ & 0.07 \\
Previous natural labor $(\mathrm{n})$ & 0 & $0.1 \pm 0.3$ & 0.18 \\
\hline
\end{tabular}




\begin{tabular}{|c|c|c|c|c|c|c|c|c|c|}
\hline \multirow{2}{*}{$\begin{array}{lr}\text { Table2 Comparison of } \\
\text { intraoperative } \\
\text { postoperative outcomes }\end{array}$} & \multicolumn{3}{|c|}{ PAS2 } & \multicolumn{3}{|c|}{ PAS3 } & \multicolumn{3}{|c|}{ PAS4-5 } \\
\hline & $\begin{array}{l}\text { Study group } \\
\qquad(\mathrm{n}=18)\end{array}$ & $\begin{array}{l}\text { Control group } \\
\qquad(\mathrm{n}=24)\end{array}$ & $P$ value & $\begin{array}{l}\text { Study group } \\
\qquad(\mathrm{n}=24)\end{array}$ & $\begin{array}{l}\text { Control group } \\
\qquad(\mathrm{n}=20)\end{array}$ & $P$ value & $\begin{array}{l}\text { Study group } \\
\qquad(\mathrm{n}=13)\end{array}$ & $\begin{array}{l}\text { Control group } \\
\qquad(\mathrm{n}=11)\end{array}$ & $P$ value \\
\hline \multicolumn{10}{|l|}{ Hysterectomy, n(\%) } \\
\hline & 0 & 0 & & 0 & $1(5.00 \%)$ & 0.93 & $2(15.38 \%)$ & $7(63.64 \%)$ & 0.03 \\
\hline Duration of the operation , $\mathrm{h}$ & $48.7 \pm 11.5$ & $45.3 \pm 11.0$ & 0.33 & $55.6 \pm 16.3$ & $48.3 \pm 12.7$ & 0.11 & $95.2 \pm 40$ & $104.6 \pm 47.2$ & 0.11 \\
\hline & & & & & & & $2812.3 \pm$ & $3204.4 \pm$ & \\
\hline Estimated blood loss (ml) & $\begin{array}{l}555.6 \pm 372.5 \\
2\end{array}$ & $518.8 \pm 362.3$ & 0.75 & $791.7 \pm 641.7$ & $783.8 \pm 741.4$ & 0.97 & 1756.7 & 1565.1 & 0.63 \\
\hline $\begin{array}{l}\text { PPH(blood loss } \geq 1000 \mathrm{ml}) \\
\mathrm{n}(\%)\end{array}$ & $(11.11 \%)$ & $3(12.50 \%)$ & 1.00 & $5(20.83 \%)$ & $5(25.00 \%)$ & 1 & $11(84.62 \%)$ & $9(81.82 \%)$ & 1.00 \\
\hline HGB $\quad(g / L)$ & $9.2 \pm 9.4$ & $7.5 \pm 6.7$ & 0.50 & $12.4 \pm 12.9$ & $15.2 \pm 12.4$ & 0.47 & $17.0 \pm 12.3$ & $26.2 \pm 12.7$ & 0.08 \\
\hline & 4 & & & & & & & & \\
\hline Transfusion rate, $\mathrm{n}(\%)$ & $(22.22 \%)$ & $4(16.67 \%)$ & 0.96 & $6(25.00 \%)$ & $7(35.00 \%)$ & 0.47 & $10(76.92 \%)$ & $11(100 \%)$ & 0.22 \\
\hline DIC, $n(\%)$ & 0 & 0 & & 0 & $1(5.00 \%)$ & 0.93 & $5(38.46 \%)$ & $9(81.82 \%)$ & 0.05 \\
\hline ICU admission, $\mathrm{h}$ & $20.7 \pm 5.9$ & $21.4 \pm 12.4$ & 0.20 & $23.1 \pm 7.8$ & $16.5 \pm 13.5$ & 0.05 & $32 \pm 11.3$ & $36.5 \pm 16.0$ & 0.43 \\
\hline Bladder repair, n (\%) & 0 & 0 & & 0 & 0 & & 0 & 0 & \\
\hline
\end{tabular}


23

\begin{tabular}{|c|c|c|c|c|c|c|c|c|c|}
\hline \multirow[t]{2}{*}{ water sac packing ,n(\%) } & $(83.33 \%)$ & $(70.83 \%)$ & 0.57 & $(95.83 \%)$ & $14(70.00 \%)$ & 0.06 & $11(84.62 \%)$ & $8(72.73 \%)$ & 0.63 \\
\hline & 16 & 15 & & 22 & & & & & \\
\hline \multirow[t]{2}{*}{8 suture,$n(\%)$} & $(88.89 \%)$ & $(62.50 \%)$ & 0.12 & $(91.67 \%)$ & $14(70.00 \%)$ & 0.14 & $12(92.31 \%)$ & $7(63.64 \%)$ & 0.14 \\
\hline & 3 & & & 10 & & & & & \\
\hline UAE , n(\%) & $(16.67 \%)$ & $2(8.33 \%)$ & 0.73 & $(41.67 \%)$ & $7(35.00 \%)$ & 0.65 & $9(69.23 \%)$ & $3(27.27 \%)$ & 0.10 \\
\hline $\begin{array}{l}\text { Maternal complications } \\
\mathrm{n}(\%)\end{array}$ & 0 & $2(8.33 \%)$ & 0.61 & $4(16.67 \%)$ & $2(10.00 \%)$ & 0.84 & $3(23.08 \%)$ & $2(18.18 \%)$ & 1.00 \\
\hline PHT , d & $6.0 \pm 2.9$ & $4.7 \pm 1.9$ & 0.09 & $5.8 \pm 2.2$ & $5.9 \pm 2.7$ & 0.89 & $9.5 \pm 6.1$ & $6.8 \pm 1.3$ & 0.18 \\
\hline
\end{tabular}

HGB: Decrease in the HGB level postoperatively

water sac packing : intrauterine water sac packing

8 suture : 8-shaped continuous suture

PHT :Postoperative hospitalization time

UAE : Uterine artery embolization 
Table 3

Neonatal outcomes and Apgar scores.

\begin{tabular}{llll}
\hline & study group $(\mathrm{n}=55)$ & control group $(\mathrm{n}=55)$ & $\mathrm{p}$ \\
\hline Birth weight, $\mathrm{g}$ & $2826.3 \pm 410.4$ & $2876.5 \pm 568.6$ & 0.59 \\
Apgar score at $1 \mathrm{~min}$ & & \\
10 & $24(43.6 \%)$ & $40(72.7 \%)$ \\
8,9 & $24(43.7 \%)$ & $10(18.2 \%)$ \\
$\leqslant 7$ & $7(12.7 \%)$ & $5(9.1 \%)$ \\
Apgar score at 5 min & & \\
10 & $38(69.1 \%)$ & $49(89.1 \%)$ \\
8,9 & $15(27.3 \%)$ & $5(9.1 \%)$ \\
$\leqslant 7$ & $3(5.5 \%)$ & $1(1.8 \%)$ \\
Apgar score at 5 min & & \\
10 & $45(81.8 \%)$ & $46(83.6 \%)$ \\
8,9 & $10(18.2 \%)$ & $9(16.4 \%)$ \\
$\leqslant 7$ & 0 & 0 \\
\hline
\end{tabular}

